Table 1 Crystallographic data for $\mathrm{Ce}_{3} \mathrm{Pt}_{5} \mathrm{Al}$ and $\mathrm{Ce}_{3} \mathrm{Pt}_{5} \mathrm{Al}_{2}$ and experimental details of the structure determination and refinement.

\begin{tabular}{l|l|l}
\hline Compound & $\mathrm{Ce}_{3} \mathrm{Pt}_{5} \mathrm{Al}$ & $\mathrm{Ce}_{3} \mathrm{Pt}_{5} \mathrm{Al}_{2}$ \\
\hline Space group & Imma & $P-1$ \\
Lattice parameters $\left(\AA{ }^{\circ}{ }^{\circ}\right)$ & $a=7.3767(4), b=13.1733(8)$, & $a=5.689(2), b=8.432(3), c=8.7103(13)$ \\
& $c=7.5884(4)$ & $\alpha=102.31(2), \beta=97.89(2), \gamma=89.10(3)$ \\
Cell volume $\left(\AA^{3}\right), Z$ & $737.41(7), 4$ & $404.3(2), 2$ \\
Calculated density $\left(\mathrm{g} / \mathrm{cm}^{3}\right)$ & 12.816 & 11.908 \\
Scan range $\theta\left(^{\circ}\right)$ & $3.85-30.00$ & $2.42-30.98$ \\
Linear absorption coefficient & $112.572 \mathrm{~mm}^{-1}$ & $102.769 \mathrm{~mm}^{-1}$ \\
Range in $h k l$ & $-8 \leq h \leq 10,-18 \leq k \leq 18$, & $-8 \leq h \leq 8,-12 \leq k \leq 11$, \\
& $-10 \leq l \leq 10$ & $0 \leq l \leq 12$ \\
Independent reflections & 597 & 2563 \\
Reflections with $I>2 \sigma(I)$ & 562 & 1875 \\
Number of refined parameters & 29 & 92 \\
Goodness of fit & 1.127 & 1.049 \\
$\mathrm{R}, \mathrm{R}_{\mathrm{w}}$ & $0.0288,0.0677$ & $0.0577,0.1422$ \\
\hline
\end{tabular}

Table 2 Atomic coordinates and equivalent isotropic displacement parameters $\left(\AA^{2}\right)$ for the structures of $\mathrm{Ce}_{3} \mathrm{Pt}_{5} \mathrm{Al}$ and $\mathrm{Ce}_{3} \mathrm{Pt}_{5} \mathrm{Al}_{2}$.

\begin{tabular}{l|l|c|c|c|c}
\hline $\mathrm{Ce}_{3} \mathrm{Pt}_{5} \mathrm{Al}$ & Atom & $x$ & $y$ & $\mathrm{Z}$ & $U_{\mathrm{eq}}$ \\
& Ce1 & 0 & $0.05036(5)$ & $0.27709(9)$ & $0.00556(19)$ \\
& $\mathrm{Ce} 2$ & 0 & $1 / 4$ & $0.62259(14)$ & $0.0074(2)$ \\
& $\mathrm{Pt} 1$ & $0.29498(5)$ & $0.10423(3)$ & $0.56604(4)$ & $0.00786(16)$ \\
& Pt2 & $1 / 4$ & $1 / 4$ & $1 / 4$ & $0.0153(2)$ \\
& Al & 0 & $1 / 4$ & $0.0276(8)$ & $0.0075(10)$ \\
\hline $\mathrm{Ce}_{3} \mathrm{Pt}_{5} \mathrm{Al}_{2}$ & Atom & $x$ & $y$ & $z$ & $U_{\mathrm{eq}}$ \\
\hline & Ce1 & $0.1459(2)$ & $0.71380(17)$ & $0.06628(13)$ & $0.0065(3)$ \\
& Ce2 & $0.2285(2)$ & $0.42124(17)$ & $0.36681(13)$ & $0.0060(2)$ \\
& Ce3 & $0.3210(2)$ & $0.01060(17)$ & $0.78986(13)$ & $0.0071(3)$ \\
& Pt1 & $0.00269(15)$ & $0.22589(11)$ & $0.57760(9)$ & $0.00550(19)$ \\
& Pt2 & $0.17583(16)$ & $0.08782(12)$ & $0.12280(9)$ & $0.0085(2)$ \\
& Pt3 & $0.39148(15)$ & $0.36000(11)$ & $0.04874(9)$ & $0.0068(2)$ \\
& Pt4 & $0.52558(15)$ & $0.21845(11)$ & $0.57544(9)$ & $0.0058(2)$ \\
& Pt5 & $0.72846(16)$ & $0.33181(12)$ & $0.31981(9)$ & $0.0082(2)$ \\
& Al1 & $0.2163(13)$ & $0.0240(10)$ & $0.3936(7)$ & $0.0078(13)$ \\
& Al2 & $0.3093(12)$ & $0.4096(9)$ & $0.7736(7)$ & $0.0063(13)$ \\
\hline
\end{tabular}

Table 3 Interatomic distances in the structure of $\mathrm{Ce}_{3} \mathrm{Pt}_{5} \mathrm{Al}$.

\begin{tabular}{|c|c|c|c|c|c|}
\hline Atom & To atom & $d, \AA$ & Atom & To atom & $d, \AA$ \\
\hline \multirow[t]{9}{*}{ Ce1 } & $2 \mathrm{Pt1}$ & $2.9999(7)$ & \multirow[t]{11}{*}{ Pt1 } & $\mathrm{Al}$ & $2.5455(17)$ \\
\hline & $2 \mathrm{Pt} 1$ & $3.0937(7)$ & & Pt1 & $2.8697(7)$ \\
\hline & $2 \mathrm{Pt} 1$ & $3.1696(6)$ & & $\mathrm{Ce} 2$ & $2.9337(4)$ \\
\hline & $2 \mathrm{Pt} 1$ & $3.2092(6)$ & & Pt1 & $2.9234(8)$ \\
\hline & $2 \mathrm{Pt} 2$ & $3.2187(6)$ & & $\mathrm{Ce} 1$ & $2.9999(7)$ \\
\hline & $\mathrm{Al}$ & $3.241(4)$ & & Pt1 & $3.0247(8)$ \\
\hline & $\mathrm{Ce} 1$ & $3.6339(14)$ & & $\mathrm{Pt} 2$ & $3.0902(4)$ \\
\hline & $2 \mathrm{Ce} 1$ & $3.7112(2)$ & & $\mathrm{Ce} 1$ & $3.0937(7)$ \\
\hline & $\mathrm{Ce} 2$ & $3.7135(10)$ & & $\mathrm{Ce} 1$ & $3.1696(6)$ \\
\hline \multirow[t]{10}{*}{$\mathrm{Ce} 2$} & $4 \mathrm{Pt} 1$ & $2.9337(4)$ & & $\mathrm{Ce} 1$ & $3.2092(6)$ \\
\hline & $\mathrm{Al}$ & $3.073(6)$ & & $\mathrm{Ce} 2$ & $3.3996(8)$ \\
\hline & $2 \mathrm{Pt} 2$ & $3.3757(9)$ & \multirow[t]{4}{*}{ Pt2 } & $2 \mathrm{Al}$ & $2.500(4)$ \\
\hline & $4 \mathrm{Pt} 1$ & $3.3996(8)$ & & $4 \mathrm{Pt} 1$ & $3.0902(4)$ \\
\hline & $2 \mathrm{Ce} 1$ & $3.7135(10)$ & & $4 \mathrm{Ce} 1$ & $3.2187(6)$ \\
\hline & & & & $2 \mathrm{Ce} 2$ & $3.3757(9)$ \\
\hline & & & \multirow[t]{4}{*}{$\mathrm{Al}$} & $2 \mathrm{Pt} 2$ & $2.500(4)$ \\
\hline & & & & $4 \mathrm{Pt} 1$ & $2.5455(17)$ \\
\hline & & & & $\mathrm{Ce} 2$ & $3.073(6)$ \\
\hline & & & & $2 \mathrm{Ce} 1$ & $3.241(4)$ \\
\hline
\end{tabular}


2.9337(4)-3.3996(8) $\AA$ for Ce2. Ten Pt-atoms build distorted pentagonal prisms around the Ce-atoms and one Al-atom caps one of the side faces of the prisms. The next-nearest neighbors are cerium atoms with a $\mathrm{Ce}-\mathrm{Ce}$ separation of $3.6339(14) \AA$ for $\mathrm{Ce} 1$ and 3.7135(10) A for Ce2.

The Pt1 atom occupies a general position $16 j$ and its coordination polyhedron with 11 apices $\left(\mathrm{Pt} 1\left[\mathrm{Ce}_{6} \mathrm{Pt}_{4} \mathrm{Al}\right]\right)$ is strongly distorted. The $\mathrm{Pt} 2$ atom occupies a $4 c$ position and its polyhedron $\left(\mathrm{Pt} 2\left[\mathrm{Ce}_{6} \mathrm{Pt}_{4} \mathrm{Al}_{2}\right]\right)$ can be described as a slightly distorted cuboctahedron.

The Al atom occupies the center of a fairly distorted trigonal prism formed by six platinum atoms with three additional $\mathrm{Ce}$ atoms capping the side faces of the prism $\mathrm{Al}\left[\mathrm{Ce}_{3} \mathrm{Pt}_{6}\right]$.

\section{b) $\mathbf{C e}_{3} \mathbf{P t}_{\mathbf{5}} \mathbf{A l}_{2}$}

The structure of $\mathrm{Ce}_{3} \mathrm{Pt}_{5} \mathrm{Al}_{2}$ was solved in the triclinic space group $P-1$ with $a=5.689(2) \AA, b=8.432(3) \AA$, $c=8.7103(13) \AA, \quad \alpha=102.31(2)^{\circ}, \quad \beta=97.89(2)^{\circ}$, $\gamma=89.10(3)^{\circ}$ (Pearson symbol $a P 20$ ) and represents a novel structure type. A space group of higher symmetry could not be evidenced using the program PLATON [17].

In the structure of $\mathrm{Ce}_{3} \mathrm{Pt}_{5} \mathrm{Al}_{2}$, all the Ce-centered polyhedra are strongly distorted with the shortest $\mathrm{Ce} 1-\mathrm{Ce} 3$ and $\mathrm{Ce} 2-\mathrm{Ce} 2$ contacts equal to 3.675(2) $\AA$

Table 4 Interatomic distances in the structure of $\mathrm{Ce}_{3} \mathrm{Pt}_{5} \mathrm{Al}_{2}$.

\begin{tabular}{|c|c|c|c|c|c|c|c|c|}
\hline Atom & To atom & $d, \AA$ & Atom & To atom & $d, \AA$ & Atom & To atom & $d, \AA$ \\
\hline \multirow[t]{12}{*}{$\mathrm{Ce} 1$} & Pt3 & $2.9538(18)$ & \multirow[t]{11}{*}{ Pt1 } & Al1 & $2.500(8)$ & \multirow[t]{9}{*}{$\mathrm{Pt} 5$} & $\mathrm{Al} 2$ & $2.481(8)$ \\
\hline & Pt2 & $3.0184(17)$ & & Al1 & $2.539(8)$ & & $\mathrm{Ce} 3$ & $2.847(2)$ \\
\hline & $\mathrm{Pt} 2$ & $3.090(2)$ & & $\mathrm{Al} 2$ & $2.549(7)$ & & Pt1 & $2.8501(13)$ \\
\hline & $\mathrm{Pt} 3$ & $3.1126(19)$ & & $\mathrm{Pt} 4$ & $2.7133(15)$ & & $\mathrm{Pt} 3$ & $2.8790(13)$ \\
\hline & $\mathrm{Pt} 3$ & $3.257(2)$ & & $\mathrm{Pt} 5$ & $2.8501(13)$ & & $\mathrm{Ce} 2$ & $2.9056(19)$ \\
\hline & Pt1 & $3.2601(15)$ & & Pt4 & $2.9768(16)$ & & Pt4 & $2.9751(12)$ \\
\hline & $\mathrm{Pt} 4$ & $3.3513(16)$ & & $\mathrm{Ce} 2$ & $3.1205(16)$ & & $\mathrm{Ce} 2$ & $2.9913(19)$ \\
\hline & $\mathrm{Al} 2$ & $3.381(7)$ & & $\mathrm{Ce} 2$ & $3.202(2)$ & & $\mathrm{Ce} 2$ & $3.0423(17)$ \\
\hline & Al1 & $3.416(8)$ & & $\mathrm{Ce} 3$ & $3.2251(18)$ & & $\mathrm{Ce} 1$ & $3.4740(15)$ \\
\hline & $\mathrm{Al} 2$ & $3.425(7)$ & & $\mathrm{Ce} 1$ & $3.2601(15)$ & \multirow[t]{11}{*}{ Al1 } & Pt1 & $2.500(8)$ \\
\hline & $\mathrm{Al} 2$ & $3.459(7)$ & & $\mathrm{Ce} 3$ & $3.6588(18)$ & & Pt2 & $2.509(6)$ \\
\hline & $\mathrm{Pt} 4$ & $3.4740(15)$ & \multirow[t]{9}{*}{$\mathrm{Pt} 2$} & Al1 & $2.509(6)$ & & $\mathrm{Pt} 4$ & $2.538(8)$ \\
\hline \multirow[t]{13}{*}{$\mathrm{Ce} 2$} & $\mathrm{Pt} 5$ & $2.9056(19)$ & & Pt3 & $2.8506(16)$ & & Pt1 & $2.539(8)$ \\
\hline & Pt3 & $2.9804(14)$ & & Pt2 & $2.888(2)$ & & Pt4 & $2.552(8)$ \\
\hline & $\mathrm{Pt} 5$ & $2.9913(19)$ & & $\mathrm{Ce} 3$ & $3.0098(19)$ & & $\mathrm{Ce} 3$ & $3.235(7)$ \\
\hline & $\mathrm{Pt} 5$ & $3.0423(17)$ & & $\mathrm{Ce} 1$ & $3.0184(17)$ & & $\mathrm{Ce} 3$ & $3.240(7)$ \\
\hline & $\mathrm{Pt} 4$ & $3.0780(17)$ & & $\mathrm{Ce} 3$ & $3.0580(14)$ & & Al1 & $3.353(14)$ \\
\hline & Pt1 & $3.1205(16)$ & & Ce1 & $3.090(2)$ & & $\mathrm{Ce} 2$ & $3.410(9)$ \\
\hline & Pt2 & $3.1330(19)$ & & $\mathrm{Ce} 2$ & $3.1330(19)$ & & $\mathrm{Ce} 1$ & $3.416(8)$ \\
\hline & Pt1 & $3.202(2)$ & & $\mathrm{Ce} 3$ & $3.1918(19)$ & & $\mathrm{Ce} 3$ & $3.447(6)$ \\
\hline & $\mathrm{Pt} 4$ & $3.276(2)$ & \multirow[t]{10}{*}{ Pt3 } & $\mathrm{Al} 2$ & $2.500(6)$ & $\mathrm{A} 12$ & $\mathrm{Pt} 5$ & $2.481(8)$ \\
\hline & Al1 & $3.410(9)$ & & $\mathrm{Al} 2$ & $2.677(8)$ & & Pt3 & $2.500(6)$ \\
\hline & $\mathrm{Al} 2$ & $3.491(7)$ & & Pt2 & $2.8506(16)$ & & $\mathrm{Pt} 4$ & $2.535(7)$ \\
\hline & $\mathrm{Al} 2$ & $3.531(7)$ & & Pt5 & $2.8790(13)$ & & Pt1 & $2.549(7)$ \\
\hline & $\mathrm{Al} 2$ & $3.532(6)$ & & $\mathrm{Ce} 1$ & $2.9538(18)$ & & Pt3 & $2.677(7)$ \\
\hline \multirow[t]{16}{*}{$\mathrm{Ce} 3$} & $\mathrm{Pt} 5$ & $2.847(2)$ & & $\mathrm{Ce} 2$ & $2.9804(14)$ & & Ce1 & $3.381(7)$ \\
\hline & $\mathrm{Pt} 2$ & $3.0098(19)$ & & Pt3 & $3.009(2)$ & & $\mathrm{Ce} 3$ & $3.397(8)$ \\
\hline & $\mathrm{Pt} 2$ & $3.0580(14)$ & & $\mathrm{Ce} 1$ & $3.1126(19)$ & & $\mathrm{Ce} 1$ & $3.425(7)$ \\
\hline & $\mathrm{Pt} 4$ & $3.1578(17)$ & & $\mathrm{Ce} 1$ & $3.257(2)$ & & $\mathrm{Ce} 1$ & $3.459(7)$ \\
\hline & $\mathrm{Pt} 2$ & $3.1918(19)$ & & $\mathrm{Ce} 3$ & $3.2988(19)$ & & $\mathrm{Ce} 2$ & $3.491(7)$ \\
\hline & Pt1 & $3.2251(18)$ & \multirow[t]{11}{*}{$\mathrm{Pt} 4$} & $\mathrm{~A} 12$ & $2.535(7)$ & & $\mathrm{Ce} 2$ & $3.531(7)$ \\
\hline & Al1 & $3.235(7)$ & & Al1 & $2.538(8)$ & & $\mathrm{Ce} 2$ & $3.532(6)$ \\
\hline & Al1 & $3.240(7)$ & & Al1 & $2.552(8)$ & & & \\
\hline & Pt3 & $3.2988(19)$ & & Pt1 & $2.7133(15)$ & & & \\
\hline & $\mathrm{Al} 2$ & $3.397(8)$ & & Pt5 & $2.9751(12)$ & & & \\
\hline & Al1 & $3.447(6)$ & & Pt1 & $2.9768(16)$ & & & \\
\hline & $\mathrm{Pt} 4$ & $3.5646(16)$ & & $\mathrm{Ce} 2$ & $3.0780(17)$ & & & \\
\hline & Pt1 & $3.6588(18)$ & & $\mathrm{Ce} 3$ & $3.1578(17)$ & & & \\
\hline & & & & $\mathrm{Ce} 2$ & $3.276(2)$ & & & \\
\hline & & & & $\mathrm{Ce} 1$ & $3.3513(16)$ & & & \\
\hline & & & & $\mathrm{Ce} 3$ & $3.5646(16)$ & & & \\
\hline
\end{tabular}


and 3.680(3) $\AA$, respectively. Though the values of the shortest $\mathrm{Ce}-\mathrm{Ce}$ contacts in the structures of $\mathrm{Ce}_{3} \mathrm{Pt}_{5} \mathrm{Al}$ and $\mathrm{Ce}_{3} \mathrm{Pt}_{5} \mathrm{Al}_{2}$ are comparable, in the structure of $\mathrm{Ce}_{3} \mathrm{Pt}_{5} \mathrm{Al}_{2}$, all three $\mathrm{Ce}$ atoms have more non-cerium neighbors (12 or 13) in their environment (see Table 3).

The five crystallographically different $\mathrm{Pt}$ sites have different coordination environments: $\mathrm{Pt} 1\left[\mathrm{Ce}_{5} \mathrm{Pt}_{3} \mathrm{Al}_{3}\right]$, $\mathrm{Pt} 2\left[\mathrm{Ce}_{6} \mathrm{Pt}_{2} \mathrm{Al}\right], \quad \mathrm{Pt} 3\left[\mathrm{Ce}_{5} \mathrm{Pt}_{3} \mathrm{Al}_{2}\right], \quad \mathrm{Pt} 4\left[\mathrm{Ce}_{5} \mathrm{Pt}_{3} \mathrm{Al}_{3}\right]$, and $\mathrm{Pt} 5\left[\mathrm{Ce}_{5} \mathrm{Pt}_{3} \mathrm{Al}\right]$.

The coordination number of the two crystallographically independent aluminum atoms in the structure of $\mathrm{Ce}_{3} \mathrm{Pt}_{5} \mathrm{Al}_{2}$ is equal to 12 . However, the $\mathrm{Al} 1$ and $\mathrm{Al} 2$ atoms have different neighbors. Five $\mathrm{Pt}$ atoms, seven $\mathrm{Ce}$ atoms and two aluminum atoms are the nearest neighbors of Al1. Al2 is surrounded by five $\mathrm{Pt}$ atoms and seven $\mathrm{Ce}$ atoms. As a result, the coordination polyhedron of $\mathrm{Al} 2$ can be described as a distorted icosahedron, whereas the coordination polyhedron of All can hardly be described in any attractive way.

The structures of $\mathrm{Ce}_{3} \mathrm{Pt}_{5} \mathrm{Al}$ and $\mathrm{Ce}_{3} \mathrm{Pt}_{5} \mathrm{Al}_{2}$ can be presented as packings of $\mathrm{Ce}$-centered polyhedra (Fig. 1).

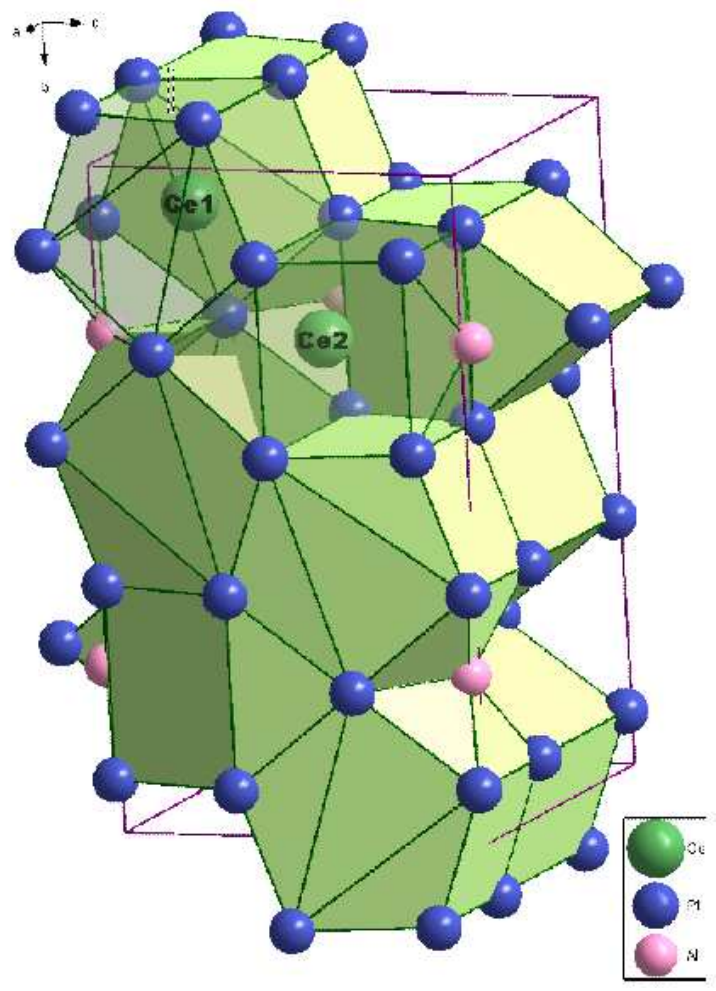

(a)

\section{Acknowledgements}

This work was supported by the RFBR projects nos. 11-03-00957a and 11-03-01191a.

\section{References}

[1] B. Xue, H. Schwer, J. Alloys Compd. 204 (1994) L25-L26.

[2] Z. Blazina, S.M. Westwood, J. Alloys Compd. 201 (1993) 151-155.

[3] C. Schank, F. Jährling, L. Luo, A. Grauel, C. Wassilew, R. Borth, G. Olesch, C.D. Bredl, C. Geibel, F. Steglich, J. Alloys Compd. 207-208 (1994) 329-332.

[4] E.V. Murashova, A.I. Tursina, N.G. Bukhan'ko, A.V. Gribanov, I.V. Chernyshev, Yu.D. Seropegin, J. Alloys Compd. 398 (2005) 100-105.

[5] A.I. Tursina, N.G. Bukhan'ko, A.V. Gribanov, V.A. Shchelkunov, Y.V. Nelyubina, Acta Crystallogr. E 61 (2005) i285-i286.

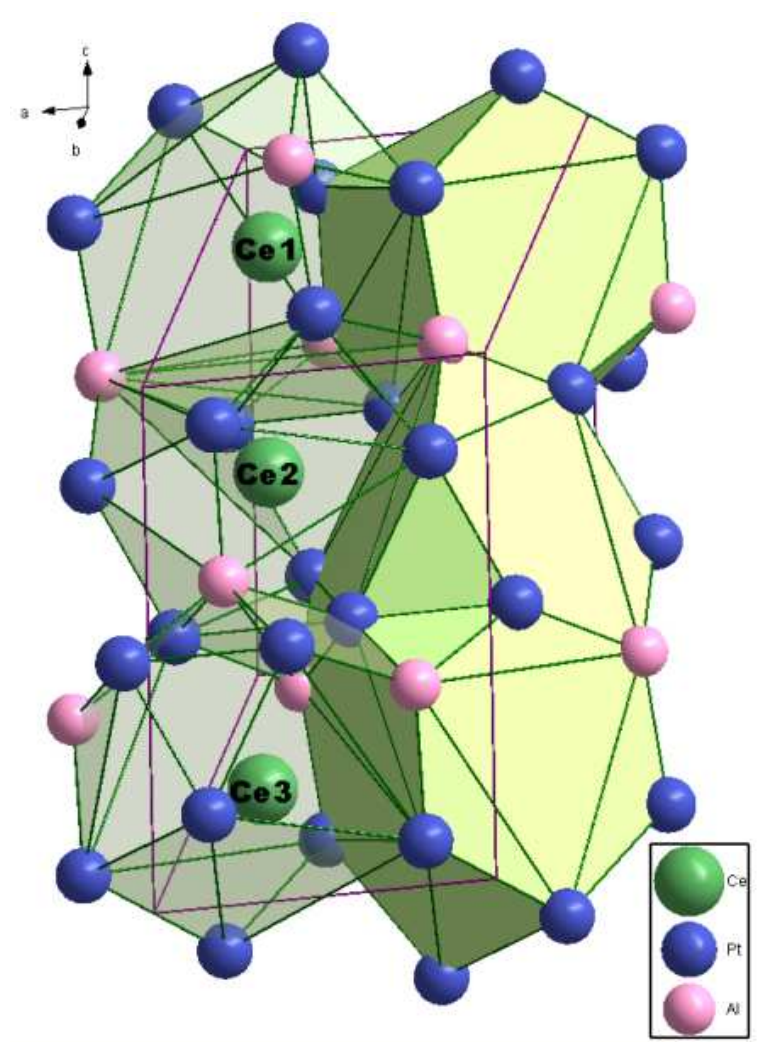

(b)

Fig. 1 Polyhedral representation of the structures of $\mathrm{Ce}_{3} \mathrm{Pt}_{5} \mathrm{Al}$ (a) and $\mathrm{Ce}_{3} \mathrm{Pt}_{5} \mathrm{Al}_{2}$ (b). 
A. Tursina et al., Crystal structures of the new intermetallics $\mathrm{Ce}_{3} \mathrm{Pt}_{5} \mathrm{Al}$ and $\mathrm{Ce}_{3} \mathrm{Pt}_{5} \mathrm{Al}_{2}$

[6] A.I. Tursina, A.V. Gribanov, N.G. Bukhan'ko, P. Rogl, Y.D. Seropegin, Chem. Met. Alloys 1 (2008) 62-66.

[7] A.I. Tursina, E.V. Murashova, H. Noël, N.G. Bukhan'ko, Y.D. Seropegin, Intermetallics 17 (2009) 780-783.

[8] A.I. Tursina, E.V. Murashova, N.G. Bukhan'ko, T. Roisnel, H. Noël, Coll. Abstr. SCTE 2008, Dresden, Germany, p. 343.

[9] G.M. Sheldrick, SADABS, University of Göttingen, Germany, 1996.

[10] L.J. Farrugia, J. Appl. Crystallogr. 32 (1999) 837-838.

[11] G.M. Sheldrick, Acta Crystallogr. A 64 (2008) 112-122.
[12] L.M. Gelato, E. Parthé, J. Appl. Crystallogr. 20 (1987) 139-143.

[13] A.V. Gribanov, Yu.D. Seropegin, O.L. Kubarev, L.G. Akselrud, O.I. Bodak, J. Alloys Compd. 317-318 (2001) 324-326.

[14] J.N. Chotard, O. Tougait, H. Noël, P. Rogl, A. Zelinskiy, O.I. Bodak, J. Alloys Compd. 407 (2006) 36-43.

[15] A. Gribanov, A. Grytsiv, E. Royanian, P. Rogl, E. Bauer, G. Giester, Y. Seropegin, J. Solid State Chem. 181 (2008) 2964-2975.

[16] S.K. Malik, D.C. Kundaliya, Solid State Commun. 127 (2003) 279-282.

[17] A.L. Spek, J. Appl. Crystallogr. 36 (2003) 7-13. 